\title{
Pectoral Muscle Segmentation in Mammograms based on Cartoon-Texture Decomposition
}

\author{
Adrian Galdran ${ }^{1,2}$, Artzai Picón ${ }^{1}$, Estibaliz Garrote $^{1}$, and David Pardo ${ }^{2}$ \\ 1 TECNALIA, Computer Vision Group. Parque Científico y Tecnológico, Edificio \\ 700, E-48160, Derio, Spain, \\ 2 Department of Applied Mathematics, Statistics, and Operational Research, \\ University of the Basque Country, Barrio Sarriena S/N, Campus de Leioa, E-48940, \\ Leioa, Spain, dzubiaur@gmail.com \\ https://sites.google.com/site/m2sigroup/
}

\begin{abstract}
Pectoral muscle segmentation on medio-lateral oblique views of mammograms represents an important preprocessing step in many mammographic image analysis tasks. Although its location can be perceptually obvious for a human observer, the variability in shape, size, and intensities of the pectoral muscle boundary turns its automatic segmentation into a challenging problem. In this work we propose to decompose the input mammogram into its textural and structural components at different scales prior to dynamically thresholding it into several levels. The resulting segmentations are refined with an active contour model and merged together by means of a simple voting scheme to remove possible outliers. Our method performs well compared to several other state-ofthe-art techniques. An average DICE similarity coefficient of 0.91 and mean Hausdorff distance of $3.66 \pm 3.23 \mathrm{~mm}$. validate our approach.
\end{abstract}

Keywords: Pectoral Muscle Segmentation, Mammographic Image Analysis, Breast Cancer Detection, Computer-Aided Diagnosis.

\section{Introduction}

Breast cancer is ranked as one of the most prevalent cancers worldwide [1], and prevention remains the most useful method to reduce its impact. In this sense, it is widely accepted that computer vision and image processing techniques can assist radiologists in mammographic image analysis tasks such as detection and characterization of breast lesions or estimation of breast density, which is often used for prediction of breast cancer risk.

A typical preprocessing step applied to a mammogram before any other image analysis method is the location of three representative landmarks, namely the breast border, the nipple, and the pectoral muscle [2]. From these, automatic pectoral muscle detection and segmentation from medio-lateral oblique view of mammographies is particularly interesting due to several factors. For example, an 
internal analysis of the pectoral muscle region may reveal the presence of axillary lymph nodes. Moreover, its presence can bias lesion detection algorithms, since its characteristics may be similar to those of abnormal tissues. Furthermore, the pectoral muscle can be useful in breast density estimation tasks: if the pectoral muscle is not properly removed, the residual pectoral region may be considered as dense tissue, producing an overestimation of the breast density.

In a mammogram, the pectoral muscle always appears as a roughly triangular region in one side of the image. Still, due to the intrinsic irregularity in anatomy and patient positions in the acquisition moment its location is highly variable, as well as its size, which may range from a few percentage of the image to half of the breast region. Besides, curvature of the pectoral muscle contour can appear as convex, concave or even a combination of both. Moreover, although humans perceive the muscle boundary as a visually continuous curve, variations in edge contrast are substantial along it. Often, the upper section of the boundary contains high contrast and is well delineated, but the lower part appears faded and blurred with fibro-glandular tissue.

Due to these and other implicit difficulties, pectoral muscle segmentation in mammograms is considered a demanding image analysis problem. As a result, a significant amount of research has been devoted to it in the last years. Based on the approximately triangular structure of the pectoral muscle, earlier algorithms relied on the Hough transform to identify its contour [3]. However, pectoral muscles may possess a considerably curved boundary, making the linearity assumption inadequate. Nevertheless, straight line fitting has been the basis for many methods that make an effort to refine initial linear approximations with different strategies. For example Kwok et al. [2] suggested Hough transform followed by cliff detection to progressively refine the obtained line into a curve that better fits the pectoral muscle boundary. In [4], the authors also detect the pectoral muscle initially as a straight line and then they look for local gradient maxima within a band enclosing it. Likewise, Kinoshita et al. [5] approximated the pectoral muscle by the longest straight line in the Radon domain.

There exist other strategies aiming to overcome the limitations related to general shape and position assumptions. To take into account texture as well as intensity cues, filtering approaches have been designed, such as the method proposed in [6], where a Gabor wavelets filter bank is applied to emphasize the pectoral muscle edge. Region growing and merging techniques have also been explored, such as in [7], where the watershed segmentation method is applied to obtain an initial over-segmentation that is refined afterwards by means of an specialized region merging algorithm.

Machine Learning techniques have also been suggested to effectively identify pixels belonging to the pectoral muscle. For instance in [8] the endpoints of the muscle contour in the vertical and horizontal directions are learnt with SVMs, and the shortest path joining both endpoints is selected as a candidate for the pectoral muscle boundary. Although these methods obtain good results, it must be taken into account that this class of techniques requires a set of seg- 
mented mammograms to train the classifiers before they can proceed to obtain a segmentation.

In this work we depart from previous approaches by building a method to segment the pectoral muscle that relies on an initial decomposition of the input mammogram into its cartoon and texture components. We apply the recently developed Rolling Guidance Filter [9] to remove the textural component of the mammography while retaining its underlying high-scale structure, including the pectoral muscle boundary. Simple connected component analysis and dynamic thresholding allow us to produce a reliable estimate of the location and contour of the pectoral muscle. These estimates are obtained pixelwise, and this generates ragged boundaries that are further processed with a generic active contour model in order to regularize them. To the best of our knowledge, this is the first time the cartoon-texture decomposition is proposed as a way to handle the variability in texture and intensity present within the pectoral muscle.

The rest of this work is structured as follows. In section II, we give the details of our approach. First, we provide an overview of the cartoon-texture decomposition problem and the Rolling Guidance Filter, the method we selected to remove textural information while preserving and emphasizing the edges of the pectoral muscle. Next we explain the dynamic thresholding and connected component analysis we perform to obtain the initial estimates of the pectoral muscle and we describe the active contour model we employ to post-process them. We then detail the obtained experimental results and comparison with other methods. We end up with a discussion on the results and some possible directions of improvement of our technique.

\section{Pectoral Muscle Detection Method}

The proposed method for pectoral muscle detection involves a multi-stage algorithm. First, a cartoon-texture decomposition is computed, followed by a simple dynamic thresholding that generates a rough segmentation feeding an active contours model to refine and regularize it. After running this process for different scale parameters, the method automatically extracts an optimal segmentation by merging the produced candidate regions. Full details about the main components of this process are provided in the remainder of the section.

\subsection{Initial Segmentation by Cartoon-Texture Decomposition}

Separating texture from geometry in natural images is a well-studied problem. Since the pioneering work of Yves Meyer [10], many researchers have proposed different methods to obtain a decomposition of an image I into the sum of a cartoon part $\mathrm{u}$, a simplified piecewise constant version of I where only the image shapes and edges appear, and a textural part $\mathrm{v}$ where the oscillating patterns lie. To obtain such a decomposition $\mathrm{I}=\mathrm{u}+\mathrm{v}$, the predominant approach is to minimize a variational formulation that tries to express I as a combination of functions lying in different functional spaces representing texture and structure. 
In [11] a comprehensive analysis of the existing variational algorithms that have been developed from the work of Meyer can be found.

Apart from the variational point of view, other approaches are possible to solve the cartoon-texture decomposition problem. If a denoising filtering process is able to keep or even enhance the edges of objects while removing noise, it can also be used to separate texture from structure. In this paper, we use such an scale-aware filtering procedure, namely, the recently developed Rolling Guidance filter [9]. This technique consists of an iterated non-linear filter that is able to effectively remove the texture component of an image at different scales while preserving edges and other general structures. The enderlying idea is to remove first the small structures of the input image, followed by an iterative edge recovery step. The first of these steps is achieved by simple Gaussian filtering:

$$
G(p)=\frac{1}{K_{p}} \sum_{q \in N(p)} \exp \left(-\frac{|p-q|^{2}}{2 \sigma_{s}^{2}}\right) \mathrm{I}(q),
$$

where $p$ and $q$ are pixels in the image, $\sigma_{s}$ denotes standard deviation, $N(p)$ is a neighborhood around pixel $p$ and $K_{p}$ is a normalization factor.

Then, a joint bilateral filtering is iteratively applied to the initial image. This procedure generates a series of iterations according to the following formula:

$$
\mathrm{J}^{t+1}=\frac{1}{K_{p}} \sum_{q \in N(p)} \exp \left(-\frac{|p-q|^{2}}{2 \sigma_{s}^{2}}-\frac{\left|\mathrm{J}^{t}(p)-\mathrm{J}^{t}(q)\right|^{2}}{2 \sigma_{r}^{2}}\right) \mathrm{I}(q),
$$

where $\sigma_{s}, \sigma_{r}$ are parameters controlling the spatial and intensity range of the filter. Along these iterations, the guidance image remains the input I. The initial $\mathrm{J}$ is the smoothed version of I obtained with Eq. (1). To obtain the subsequent iterations, we perform a smoothing of the input image I guided by the structure present at the previous iteration $J^{t}$. Eq. (2) smoothes the original image guided by the structure underlying in the $\mathrm{J}^{t}$ iteration, yielding a new iteration $\mathrm{J}^{t+1}$ in which small structures are not present, but a part of the original large scale structure is retrieved. This process has the ability to enhance the so-called virtual edges. These are edges that do not come from large gradients, but rather from different large scale image information. An example of the ability of the Rolling Guidance Filter to emphasize this kind of information is shown in Fig. 1.

\subsection{Dynamic Thresholding and Connected Component Analysis}

After extracting the structural part from the input mammogram, we threshold it at multiple values supplied by a dynamic thresholding procedure. Quantization of the cartoon part of the mammogram at these values provides a piecewise constant image in which the upper leftmost connected component roughly represents the pectoral muscle, see Fig. 2. To ensure a correct segmentation, we employ several number of quantization levels, specifically 3 to 5 levels. In each quantized image, we pick the largest upper leftmost connected component that passes a simple triangularity test. This test is an easy verification of the triangular arrangement 


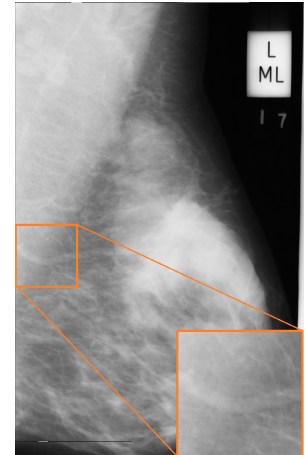

(a)

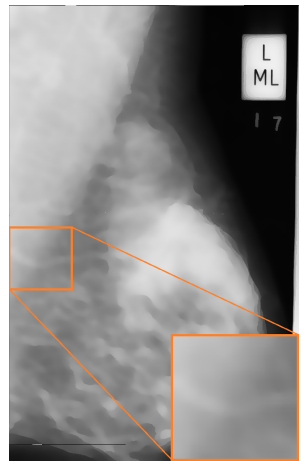

(b)

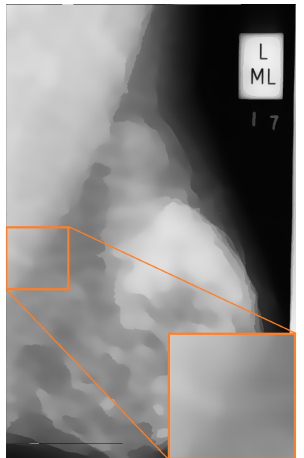

(c)

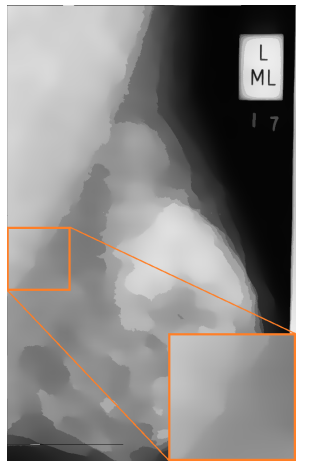

(d)

Fig. 1. a) Original mammogram, b)-d) Mammogram filtered at different scales $\sigma_{s} \in$ $\{6,10,20\}$. Notice how the border of the muscle is hardly distinguishable by its intensity in a close look, although its presence is intuitively obvious. As the scale of the removed structures increases that virtual edge is emphasized, while vessels and other structures lying within the pectoral muscle are blended together and eventually disappear.

of pixels: sample lines of the segmentation are scanned from the top to the bottom of the image. If a sample line contains more pectoral muscle pixels than the next one we reject that candidate segmentation. Equivalently, the number of pectoral muscle pixel per line must be a decreasing function for the segmentation to be accepted. Finally, we select the output with the largest area.

\subsection{Active Contours Segmentation Refinement}

The segmentations produced so far already supply a good approximation of the pectoral muscle. However, we have experimentally observed a general tendency to underestimate the real pectoral muscle area. Moreover, the contours of the regions appear ragged, due to the pixel-wise cartoon-texture decomposition. To regularize the border of the segmentation, as well as to recover part of the missed region, we perform an active contours-based refinement process. This is carried out by means of the popular Chan-Vese model for image segmentation [12]. The energy this model aims to minimize is given by:

$$
\begin{aligned}
\mathrm{F}\left(c_{1}, c_{2}\right)= & \mu \cdot \operatorname{length}(C)+\lambda_{1} \iint_{\text {in }(C)}\left|\mathrm{I}(x, y)-c_{1}\right|^{2} d x d y \\
& +\lambda_{2} \iint_{\text {out }(C)}\left|\mathrm{I}(x, y)-c_{2}\right|^{2} d x d y
\end{aligned}
$$

where $\mu, \lambda_{1}, \lambda_{2}$ are fixed parameters and $C$ is a closed curve that evolves over the image I : $\Omega \rightarrow[0,255]$, being $\Omega$ the domain of the image. This curve encloses an inner region, denoted by $i n(C)$, and the outer region is defined as out $(C)=\Omega-i n(C)$. The constants $c_{1}, c_{2}$ represent the average of the image 


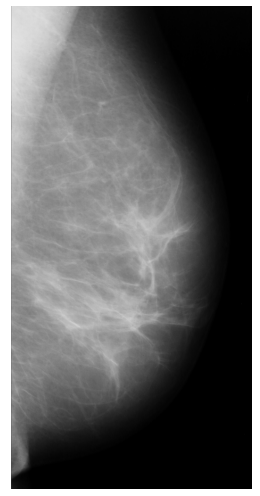

(a)

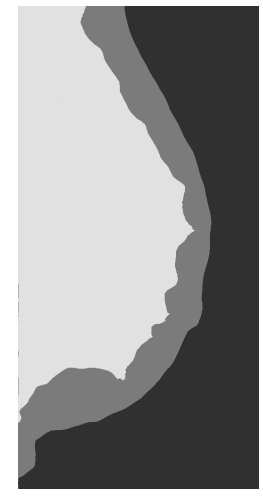

(b)

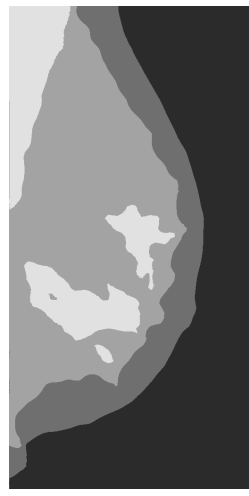

(c)

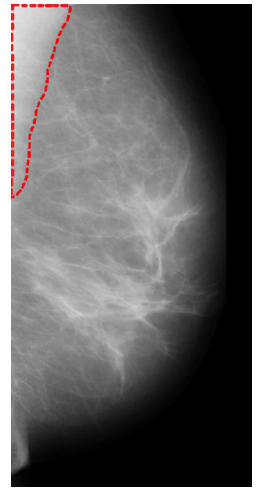

(d)

Fig. 2. a) Original mammogram b) Result after filtering at scale $\sigma_{s}=6$, dynamic thresholding with 3 levels and quantization c) Result after filtering at scale $\sigma_{s}=6$, dynamic thresholding with 4 levels and quantization e) Final output of our method.

intensity inside and outside $C$ respectively. Thus, they depend on $C$ and change as the curve evolves. This functional is minimized when these average intensities are as different as possible, while respecting the constraint on keeping the length of $C$ relatively low, imposed by the first term in Eq. (3). The initial contour is given in our case by the border of the segmentation generated in the previous subsection. This segmentation is iteratively grown in the directions that minimize Eq. (3). The low length constraint on the boundary of the segmentation implies that the border will remain simple, solving this way the issue about the ragged high-curvature contours remarked above. Let us underline that the evolution of the active contour takes place on the correspondent cartoon part of the decomposition of the input mammography, rather than on the latter.

\subsection{Multi-scale decomposition and merged segmentation}

Since texture is a scale-dependent concept, there is no unique possible cartoontexture decomposition of an image. A texture seen at a close inspection is a group of well-contrasted objects, such as leaves or bricks. This structure can be considered as cartoon in a low-scale processing and texture from a large-scale point of view. Thus, the scale at which an image must be processed to suppress every texture from the cartoon part comes with some subjectivity. To make our approach robust, we run the Rolling Guidance Filter for different realizations of the scale parameter $\sigma_{s}$ to remove texture at different scales. This generates a set of segmentations that we merge by means of a simple voting scheme in which a pixel is considered to be part of the pectoral muscle if more than one of the produced segmentations contains it. This allows us to get rid of spurious outliers that may appear when removing texture with only one scale parameter. The full computational procedure of the proposed method is outlined below. 


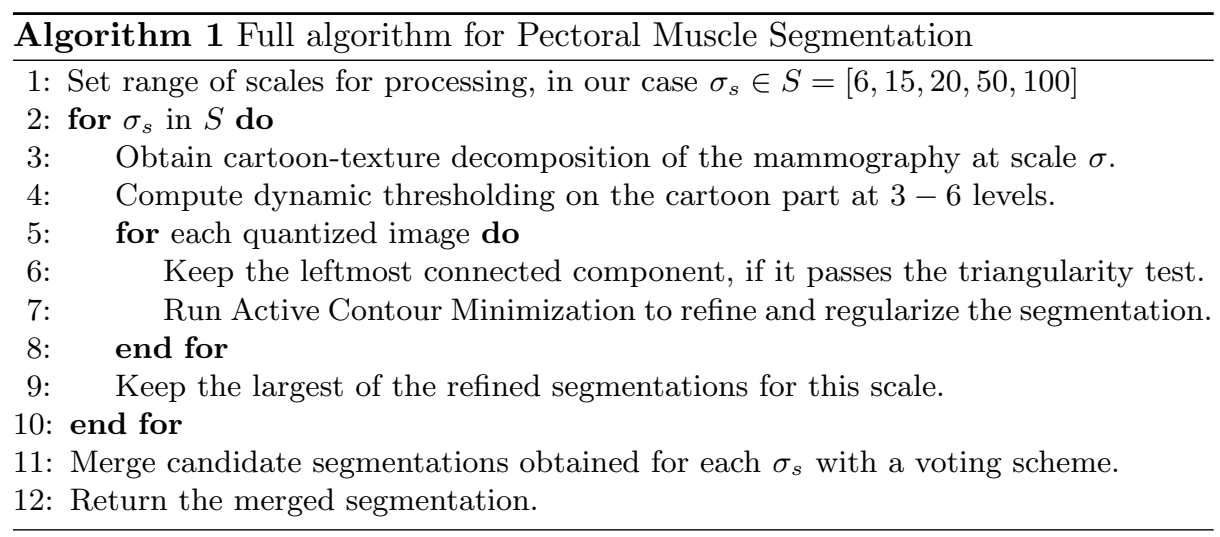

\section{Experimental Results and Discussion}

We have tested our method on a subset of the mini-MIAS database consisting of 84 mammographies. Ground-truth supplied by expert radiologists was provided in [6] and has been widely used in the literature for performance comparison.

In terms of general behavior, our method seems to be effective and robust, obtaining an average DICE coefficient of similarity 0.91 between the generated segmentations and the available ground-truth. Another popular index to compare pectoral muscle segmentation methods is the Hausdorff distance, that reflects the geometric distance between two sets $A, B$, given by:

$$
h(A, B)=\max _{a \in A} \min _{b \in B} \operatorname{dist}(a, b),
$$

where $\operatorname{dist}(a, b)$ is the Euclidean distance between two points $a, b$. Another common performance measure pectoral muscle segmentation is the average rate of false positives (FP) and false negatives (FN) found in a segmentation. Both of these coefficients, as well as the average Hausdorff distance, for our method and the techniques suggested in [6], [7], [4], [8], and [5] are displayed in Table 3.

Table 1. Error Analysis - Hausdorff distance, mean FP and mean FN

\begin{tabular}{lcccccc}
\hline \multicolumn{1}{c}{ Methods } & {$[6]$} & {$[7]$} & {$[4]$} & {$[8]$} & {$[5]$} & Ours \\
\hline Hausdorff distance - Mean & 3.84 & 3.85 & 3.84 & 2.49 & 12.45 & $\mathbf{3 . 6 6}$ \\
Hausdorff distance - StDev & 1.73 & 1.07 & 3.85 & 0.99 & 22.96 & $\mathbf{3 . 2 3}$ \\
Mean FP & 0.58 & 0.85 & 0.42 & - & 0.89 & $\mathbf{0 . 2 7}$ \\
Mean FN & 5.77 & 4.88 & 6.71 & - & 9.13 & $\mathbf{5 . 7 5}$ \\
\hline
\end{tabular}

We see that our method slightly outperforms the other techniques. Only [8] reports better performance. Yet, this method relies on the availability of a training set. Also, classifiers running over mammographies acquired with a given system may produce errors if applied to mammograms coming from another one. 


\section{Conclusions and Future Research}

We have presented a new pectoral muscle segmentation method that employs cartoon-texture decomposition to overcome the variability in texture and intensity that hinders the performance of other techniques. Experimental results support our approach, showing a good comparison against other recent methods. Furthermore, our method implements a generic active contour method to refine the produced segmentation. Further improvements can be achieved by designing a specialized active contour model accounting for the specificities of the problem.

The cartoon-texture decomposition may also be an effective tool to handle other mammographic image analysis problems, such as breast-skin line detection. We are currently exploring this an other applications.

\section{References}

1. Bray, F., Ren, J.S., Masuyer, E., Ferlay, J.: Global estimates of cancer prevalence for 27 sites in the adult population in 2008. Int. J. Cancer 132(5) (2013) 1133-1145

2. Kwok, S.M., Chandrasekhar, R., Attikiouzel, Y., Rickard, M.: Automatic pectoral muscle segmentation on mediolateral oblique view mammograms. IEEE Transactions on Medical Imaging 23(9) (2004) 1129-1140

3. Karssemeijer, N.: Automated classification of parenchymal patterns in mammograms. Phys. Med. Biol. 43(2) (1998) 365

4. Chakraborty, J., Mukhopadhyay, S., Singla, V., Khandelwal, N., Bhattacharyya, P.: Automatic detection of pectoral muscle using average gradient and shape based feature. J Digit Imaging 25(3) (2012) 387-399

5. Kinoshita, S.K., Azevedo-Marques, P.M., Pereira, R.R., Rodrigues, J.A.H., Rangayyan, R.M.: Radon-domain detection of the nipple and the pectoral muscle in mammograms. J Digit Imaging 21(1) (2008) 37-49

6. Ferrari, R., Rangayyan, R., Desautels, J., Borges, R., Frere, A.: Automatic identification of the pectoral muscle in mammograms. IEEE Transactions on Medical Imaging 23(2) (2004) 232-245

7. Camilus, K.S., Govindan, V.K., Sathidevi, P.S.: Pectoral muscle identification in mammograms. J Appl Clin Med Phys 12(3) (2011) 3285

8. Domingues, I., Cardoso, J.S., Passarinho, P., Cardoso, M.J.: Pectoral muscle detection in mammograms based on the shortest path with endpoints learnt by SVMs. Conf Proc IEEE Eng Med Biol Soc 2010 (2010) 3158-3161

9. Zhang, Q., Shen, X., Xu, L., Jia, J.: Rolling guidance filter. In Fleet, D., Pajdla, T., Schiele, B., Tuytelaars, T., eds.: ECCV 2014. Number 8691 in Lecture Notes in Computer Science. Springer International Publishing (2014) 815-830

10. Meyer, Y.: Oscillating Patterns in Image Processing and Nonlinear Evolution Equations: The Fifteenth Dean Jacqueline B. Lewis Memorial Lectures. American Mathematical Society, Boston, MA, USA (2001)

11. Buades, A., Le, T., Morel, J.M., Vese, L.: Fast cartoon + texture image filters. IEEE Transactions on Image Processing 19(8) (2010) 1978-1986

12. T.F. Chan, L.A. Vese: Active contours without edges. IEEE Transactions on Image Processing 10(2) (2001) 266-277 